\title{
Influence of Covariates on Involved Lymph Nodes in Primary Breast Cancer Patients: Mixture Distribution Zero-Inflated Modeling Methodological Framework
}

Madiha Liaqat

University of the Punjab

Shahid Kamal

University of the Punjab

Florian Fischer ( $\square$ florian.fischer1@charite.de)

Charite Universitatsmedizin Berlin https://orcid.org/0000-0002-4388-1245

Nadeem Zia

Mayo Hospital Lahore

\section{Research}

Keywords: Oncology, Mamma cancer, Nodal involvement, Count model, Zero-Inflation

Posted Date: September 10th, 2020

DOI: https://doi.org/10.21203/rs.3.rs-62928/v1

License: (c) (i) This work is licensed under a Creative Commons Attribution 4.0 International License.

Read Full License 


\title{
Influence of Covariates on Involved Lymph Nodes in Primary Breast Cancer Patients: Mixture Distribution Zero-Inflated Modeling Methodological Framework
}

\author{
Madiha Liaqat ${ }^{1}$, Shahid Kamal ${ }^{2}$, Florian Fischer ${ }^{3,4}$, Nadeem Zia ${ }^{5}$ \\ ${ }^{1}$ College of Statistical and Actuarial Sciences (CSAS), University of the Punjab, Lahore, Pakistan \\ ${ }^{2}$ College of Statistical and Actuarial Sciences (CSAS), University of the Punjab, Lahore, Pakistan \\ ${ }^{3}$ Institute of Public Health, Charité - Universitätsmedizin Berlin, Berlin, Germany \\ ${ }^{4}$ Institute of Gerontological Health Services and Nursing Research, Ravensburg-Weingarten University of Applied \\ Sciences, Weingarten, Germany \\ ${ }^{5}$ Department of Oncology and Radiotherapy, Mayo Hospital, Lahore, Pakistan
}

\section{Corresponding author:}

Dr. Florian Fischer

Charité - Universitätsmedizin Berlin

Institute of Public Health

Charitéplatz 1

10117 Berlin

E-Mail: florian.fischer1@ charite.de 


\section{Abstract}

2 sample.

Background: Involvement of lymph nodes has been an integral part of breast cancer prognosis and survival. This study aimed to explore factors influencing on the number of auxiliary lymph nodes in women diagnosed with primary breast cancer by choosing an efficient model to assess excess of zeros and over-dispersion presented in the study population.

Methods: The study is based on a retrospective analysis of hospital records among 5,196 female breast cancer patients in Pakistan. Zero-inflated Poisson and zero-inflated negative binomial modeling techniques are used to assess the association between under-study factors and the number of involved lymph nodes in breast cancer patients.

Results: The most common breast cancer was invasive ductal carcinoma (54.5\%). Patients median age was 48 years, from which women aged 46 years and above are the majority of the study population (64.8\%). Examination of tumors revealed that over 2,662 (51.2\%) women were ER-positive, 2,652 (51.0\%) PR-positive, and 2,754 (53.0\%) were Her2.neu-positive. The mean tumor size was $3.06 \mathrm{~cm}$ and histological grade $1(\mathrm{n}=2021,38.9 \%)$ was most common in this

The model performance was best in the zero-inflated negative binomial model. Findings indicate that most factors related to breast cancer have a significant impact on the number of involved lymph nodes. Age is not contributed to lymph node status. Women having a larger tumor size suffered from greater number of involved lymph nodes. Tumor grades 11 and 111 contributed to higher numbers of positive lymph node.

Conclusions: Zero-inflated models have successfully demonstrated the advantage of fitting count nodal data when both "at-harm" (lymph node involvement) and "not-at-harm" (no lymph node involvement) groups are important in predicting disease on set and disease progression. Our analysis showed that ZINB is the best model for predicting and describing the number of involved nodes in primary breast cancer, when overdispersion arises due to a large number of patients with no lymph node involvement. This is important for accurate prediction both for therapy and prognosis of breast cancer patients.

Keywords: Oncology, Mamma cancer, Nodal involvement, Count model, Zero-Inflation 


\section{Background}

33 Breast cancer, a commonly diagnosed malignant cancer entity in females, represents a major

34 public health issue worldwide [1]. Previous studies have shown large absolute numbers of 35 incident breast cancer cases in developing countries, in which abnormal growth starts in breast 36 tissues with the risk of spreading to other body parts [2]. This malignancy is classified into two 37 major types, ductal and lobular carcinoma: Ductal carcinoma - which most breast cancers belong 38 to - starts in the ducts; lobular carcinoma starts in the milk-producing parts of the breast 39 (lobules). Significant prognostic factors of poor survival are higher age, nodal involvement, 40 higher tumor grade, advanced clinical stage, greater tumor size, and metastasis [3].

41 Although new tumor markers have been identified and different tests are recommended, the 42 condition is not good for lower economic countries [4]. Due to lack of funding, the tumor 43 diagnosis and prognosis in public hospitals in developing countries is still limited to lymph node 44 status, tumor size, and grade [4,5], also, the presence or absence of auxiliary lymph nodes has 45 been recognized as an important predictor of breast cancer risk. Studies showed node-positive 46 patients had lower survival rates than node-negative ones [6]. Furthermore, a higher number of 47 positive lymph node involvement contributes to an increased risk of complications [7-9]. Many 48 studies show the association between various factors and the progression of breast cancer; all of 49 them highlight the importance of lymph node involvement in breast carcinoma $[10,11]$.

50 For breast cancer prediction, modeling of non-negative whole integers is done through 51 generalized linear modeling framework, by accounting non-normal response average linkage to 52 the predictors. From the exponential family distributions, any distribution of projection of non53 negative count response is used [12]. The number of involved lymph nodes falls under the 54 category of count data, which cannot be generally approximated by a normal distribution. In the 
55 context of count response variable, Poisson, negative binomial, Poisson log-normal and other 56 mixtures of distributional models are available [13-15]. Among the negative binomial and

57 Poisson regression models, binomial is mostly applied to avoid observed heterogeneity and 58 clustering, while Poisson count model has the limitations of equal mean and variance in real59 world scenarios $[16,17]$.

60 In epidemiological investigations, excessive zeros are a special case of count data analysis, 61 which cannot be applied through conventional count techniques. Zero-inflated distributional 62 modeling has proved usefulness in this regard, by dividing the regime into zeros and non-zeros. 63 These divided regions mostly called perfect and imperfect states, respectively [18]. A large 64 variety of literature is available on zero-inflated response treated with zero-inflated Poisson (ZIP) 65 or zero-inflated negative binomial (ZINB) modeling techniques [19-24].

66 Lymph node involvement in breast cancer is strongly associated with many factors. Although 67 count models are suitable for modeling lymph node counts, but distributions of lymph node 68 counts are characterized by a large number of zeroes, when there is no lymph node involvement 69 at the initial diagnostic stage of breast cancer, that's why zero inflated modeling framework has 70 replaced by count modeling to handle such "excess zeros". In studies where both excess zeros 71 and over-dispersion occurs, negative binomial zero-inflated models are applied to get efficient 72 estimates of parameters, rather than inflated Poisson [25]. Another common issue arises in 73 understanding and projecting excessive zeros in studies so an appropriate model can be chosen to 74 assess excessive zeros in the first place, by considering false negative rate [26].

75 This study aims to quantify the risk of lymph node involvement in women diagnosed with 76 primary breast cancer, by applying descriptive statistics and other tests to check excessive zeros 77 in the data set. Complete modeling methodology is presented and applied to study breast cancer 
78 disease and develop better intervention strategies related to such data type. For doing so, we used

79 retrospective data from hospital records of breast cancer patients in Pakistan.

80

\section{$81 \quad$ Methods}

\section{Study design}

83 This study is based on a retrospective analysis of data from 5,196 primary breast cancer women 84 who registered at Mayo hospital Lahore, Pakistan, from 2013 to 2019. This data taken from 85 hospital records include information about the age at diagnosis, cancer type, tumor size, 86 histological grade, and molecular markers (ER, PR, Her2.neu). The number of involved lymph

87 nodes was taken as the response variable. Complete information of predictors and response were 88 available for all selected cases. Exclusion criteria were incomplete information, patients who had 89 a secondary tumor or had metastasis from other organs to the breast at the time of registration, 90 unknown pathological nodal status $(\mathrm{Nx})$, immeasurable primary tumor (Tx), and Paget's disease

91 of the nipple without tumor. The association between the understudy factors mentioned above 92 and the number of involved nodes has been assessed using inflated count modeling.

\section{Modeling framework}

95 By representing count lymph nodes, the response variable $Y_{j}=1,2,3, \ldots, n$ has a probability mass 96 function (PMF) of Poisson distribution with parameter $\theta$,

$97 \quad f\left(y_{j}, \lambda_{j}\right)=\frac{e^{-\lambda_{j}} \lambda_{j}^{y_{j}}}{y_{j} !} \quad y_{j}=0,1,2,3, \ldots$

98 In this scenario, likelihood function takes the form

$99 \quad l(\lambda)=\ln (\lambda ; y)=\sum_{j=1}^{n}\left\{y_{j} \ln \left(\lambda_{j}\right)-\lambda_{j}-\ln \left(y_{j} !\right)\right\}$ 
100 The function $\ln \left(\lambda_{j}\right)=\eta_{j}=x_{j}{ }^{T} \alpha$ linked $Y_{j}$ and matrix of explanatory variables $X$ [12].

101 Poisson distribution has conditions of independent events and equality of conditional mean and

102 variance [13].

103 If non-negative whole integer response follows negative binomial distribution the function is 104 given by,

$105 f\left(y_{j} ; \lambda_{j}, \tau\right)=\frac{\Gamma\left(y_{j}+\frac{1}{\tau}\right)}{\Gamma\left(\frac{1}{\tau}\right) y_{j} !}\left(1+\tau \lambda_{j}\right)^{-\frac{1}{\tau}}\left(1+\frac{1}{\tau \lambda_{j}}\right)^{-y_{j}}, y_{j}=0,1,2,3, \ldots$.

106 Mean and variance are $\theta_{j}$ and $\theta_{j}\left(1+\tau \theta_{j}\right)$, respectively. Being a dispersion parameter $\tau \rightarrow 0$

107 negative binomial becomes a Poisson distribution [14].

108 Many fields of research involve excess zero counts Such data is known as zero-inflated data, in 109 which the population under study is divided into two latent classes, with most observations being 110 zero. Researchers have developed methods to analyze excessive zeroes data in various research 111 disciplines such as agriculture, ecology, economy, health, tourism, transportation, manufacturing, 112 and others. Zero-inflation has a two-stage modeling framework, where numbers are modeled 113 through count distribution and observations at zero through binomial realization [25-28].

114 Lambert (1992) proposed the theory and application of zero-inflated Poisson (ZIP) modeling by 115 a two-way process, first excess zeros and second zeros occur in Poisson distribution [19]. ZIP 116 data has the general form,

$117 \quad Y_{j} \sim\left\{\begin{array}{lll}0 & \text { probability } & \pi_{j} \\ \operatorname{Poisson}\left(\lambda_{j}\right) & \text { probability } & 1-\pi_{j}\end{array}\right.$

118 Here, $Y_{j}, j=1,2,3, \ldots, n, \pi_{j}$ is the probability of extra zeros while $\lambda_{j}$ is the average of non-extra 119 zeros subpopulation. 
120 It yields two states mixture distribution with PMF,

$121 \operatorname{Pr}\left(Y_{j}=y_{j}\right)=\left\{\begin{array}{l}\pi_{j}+\left(1-\pi_{j}\right) e^{-\lambda_{j}}, y_{j}=0 \\ \left(1-\pi_{j}\right) \frac{e^{-\lambda_{j}} \lambda^{y_{j}}}{y_{j} !}, y_{j}>0\end{array}\right.$

122 Parameters $\pi_{\mathrm{j}}$ and $\lambda_{j}$ are defined as $\operatorname{logit}\left(\pi_{j}\right)=Z_{j} \gamma$ and $\log \left(\lambda_{j}\right)=X_{j} \alpha$, where

$123 \gamma=\left(\gamma_{1}, \gamma_{2}, \gamma_{3}, \ldots, \gamma_{p 1}\right)^{\prime}, \quad \alpha=\left(\alpha_{1}, \alpha_{2}, \alpha_{3}, \ldots, \alpha_{p 2}\right)^{\prime}$ and $Z_{j}, X_{j}$ are $\left(1 \times p_{1}\right)$ and $\left(1 \times p_{2}\right)$ vectors of

124 predictors for the $j^{\text {th }}$ unit. For estimation purpose, the likelihood for ZIP has the form,

$125 l=l(\theta, \pi ; y)=\sum_{j}\left\{I_{\left(y_{j=0}\right)}\left[\ln \left(\pi_{j}+\left(1-\pi_{j}\right) e^{-\lambda_{j}}\right]+I_{\left(y_{j>0}\right)}\left[\ln \left(1-\pi_{j}\right)-\lambda_{j}+y_{j} \ln \lambda_{j}-\ln \left(y_{j} !\right)\right]\right\}\right.$

126 Where, $I($.$) is the event indicator variable.$

127 Inflated negative binomial [29] has the probability density function (PDF),

$128 p\left(Y_{j}=y_{j}\right)=\left\{\begin{array}{l}\pi_{j}+\left(1-\pi_{j}\right)\left(1+\tau \lambda_{j}\right)^{-\frac{1}{\tau}}, y_{j}=0 \\ \left(1-\pi_{j}\right) \frac{\Gamma\left(y_{j}+\frac{1}{\tau}\right)}{y_{j} ! \Gamma\left(\frac{1}{\tau}\right)}\left(1+\tau \lambda_{j}\right)^{-\frac{1}{\tau}}\left(1+\frac{1}{\tau \lambda}\right)^{-y_{j}}, y_{j}>0\end{array}\right.$

129

$l=l\left(\tau, \lambda_{j}, \pi_{j} ; y_{j}\right)=\sum_{j=1}^{n}\left\{I_{\left(y_{j}=0\right)} \ln \left(\left(\pi_{j}+\left(1-\pi_{j}\right)\left(1+\tau \lambda_{j}\right)^{-\frac{1}{\tau}}\right)+I_{\left(y_{j}>0\right)} \ln \left(\left(1-\pi_{j}\right) \frac{\Gamma\left(y_{j}+\frac{1}{\tau}\right)}{y_{j} ! \Gamma\left(\frac{1}{\tau}\right)}\left(1+\tau \lambda_{j}\right)^{-\frac{1}{\tau}}\left(1+\frac{1}{\tau \lambda_{j}}\right)^{-y_{j}}\right)\right\}\right.$

130

132 As our data set has covariates, the ZIP likelihood has the form, 
135 Similarly, the ZINB likelihood with covariates is given by,

$136 l=l\left(y_{j} \mid x_{j}, z_{j}\right)$

137

$=\sum_{j=1}^{n}\left\{I_{\left(y_{j}=0\right)}\left[\ln \left(\exp \left(z_{j}^{T} y\right)+\left(1+\tau \exp \left(x_{j}^{T} \alpha\right)^{-\frac{1}{\tau}}\right)\right]+I_{\left(y_{j}>0\right)} \sum_{j=1}^{n}\left[j \sum_{k=1}^{y_{j}} \ln \left(\tau y_{j}-\tau k+1\right)-\ln \left(1+\exp \left(\exp \left(z_{j}^{T} y\right)\right)\right)-\ln \left(y_{j} !\right)-\left(y_{j}+\frac{1}{\tau}\right) \ln \left(1+\tau \exp \left(x_{j}^{T} \alpha\right)\right)+y_{j} x_{j}^{T} \alpha\right]\right\}\right.$

139 Estimates are obtained by taking first and second derivatives with respect to unknown 140 parameters.

141 We applied the approach to model the effect of different factors related to breast cancer on the 142 number of involved lymph nodes. The covariates found to be significant in univariate analysis 143 with any of the regressions were included in all the regression models to maintain the 144 comparative findings. We first applied Poisson and negative binomial count models and then ZIP 145 and ZINB models. The Vuong test was performed to check inflated models' improvement over 146 standard parametric count models [28,30]. The general statistical model has the form,

147 Count $($ LymphNodes $)=\alpha_{0}+\alpha_{1}$ TumorType $+\alpha_{2}$ Age $+\alpha_{3}$ TumorGrade $+\alpha_{4} E R+$

$148 \quad \alpha_{5} P R+\alpha_{6}$ Her2.neu $+\alpha_{7}$ TumorSize

150 Where count is the response variable (number of involved lymph nodes), which can follow many 151 distributions, like Poisson, Quasi-Poisson, negative binomial and others. Here we applied two 152 distributional count models: Poisson and negative binomial. For the Vuong test [31], we assumed 153 Poisson and negative binomial regression models, while for the binary component of zero154 inflated $2^{7}=128$ different choices occurred. 
155 Zero inflated models reduced bias in our estimates by accounting non-normality due to 156 occurrence of large number of zeroes in our data set. Also, Akaike information criterion (AIC) 157 was criteria used to check the performance of zero-inflated models [32]:

$158 \quad A I C=-2 \ln L+2 p$

159 Where $L$ denotes the fitted $\log$ likelihood and $p$ the number of parameters, AIC is highly 160 recommended model selection criteria as it takes into account both goodness of fit and 161 complexity of the model.

162 For all analyses, the level of significance was chosen at 5\%. R 3.6.2 package was used for data 163 analysis [33].

165 Results

166 Sample characteristics and lymph node involvement

167 Table 1 shows the characteristics of female breast cancer patients, which may act as predictive 168 factors on the number of lymph nodes. The tumor is divided into invasive ductal carcinoma $169(n=2,832 ; 54.5 \%)$ and other types $(n=2,364 ; 45.5 \%)$. The median age at diagnosis was 48 and it 170 was divided into three categories $\leq 35$ years $(n=736,14.2 \%), 36-45$ years $(n=1,092 ; 21.0 \%)$, and $171 \geq 46$ years $(n=3368 ; 64.8 \%)$. The patients were almost equally distributed among the histological 172 grades. About half of the patients were ER positive (51.2\%), PR positive (51.0\%), and Her2.neu 173 positive $(53.0 \%)$. The majority of the patients $(70.2 \%)$ had a tumor size between 2 and $4.9 \mathrm{~cm}$. 174

175 - Table 1 176 
177 The plot of nodal frequency at each count is shown in Figure 1. Overall, 2,406 breast cancer 178 patients $(46,3 \%)$ had no lymph node involved, suggested inflated zero models. Among the 2,790 179 patients with positive nodes, factors are distributed among three counting categories 1-2 $180 \quad(n=656), 3-4(n=1324)$, and $\geq 5(n=810)$ of involved nodes (Table 2$).$

181

182 - Figure 1 -

183 - Table 2 -

\section{Zero-inflation modeling}

186 Zero inflated Poisson (ZIP) and negative binomial (ZINB) demonstrated significant $(\mathrm{p}<0.05)$

187 association of tumor grade, ER, PR and tumor size with counts of involved nodes, while tumor

188 type, tumor grade, ER, PR, Her2.neu and tumor size have influence on zero excessive counts of 189 no involvement of nodes (Table 3), because of not having prior information about predictors 190 which might have strong association with positive nodes and/or excessive zeroes, we take same 191 covariates on both components of two distributions mixture inflated models. Results showed 192 estimated coefficients, standard errors and associated p-values for count and zero parts for ZIP 193 and ZINB.

195 - Table 3 -

197 Vuong test [31], as a suitable suggested approach for both nested and non-nested models, favors 198 between Poisson count and ZIP the latter (Z-statistic=-26.917, $\mathrm{p}<0.001)$, and between negative 199 binomial and ZINB also the latter one (Z-statistic=-22.286, $\mathrm{p}<0.001)$. The model performance 
200 based on AIC indicated that among linear (AIC=23710.43), counts (Poisson (AIC=20777.19);

201 Negative Binomial (AIC =18773.01)) and zero inflated (ZIP (AIC=16658.36); ZINB

202 (AIC=16559.15)) the ZINB model provides the best fit to the data, as reference of lower AIC

203 value [32] (Table 4).

204

205 -Table $4-$

206

207 Due to the better fit of the ZINB model, these modeling results on factors significantly associated

208 with nodal involvement are presented (Table 5). According to this, patients with a higher tumor

209 grade have a larger number of positive lymph nodes, although strongly positive associated for

210 grade $111(\mathrm{OR}=1.323,95 \% \mathrm{CI}: 1.248-1.402)$ and $11(\mathrm{OR}=1.002,95 \% \mathrm{CI}: 0.944-1.064)$

211 compared to grade 1 . Patients being ER-negative (OR=0.952, 95\% CI: 0.913-0.993) and PR-

212 negative $(\mathrm{OR}=0.897,95 \% \mathrm{CI}$ : 0.856-0.939) have a lower likelihood for involved lymph nodes.

213 Larger tumor sizes have positive association with a greater number of positive lymph nodes

214 involved at primary diagnosed breast cancer in Pakistani women. When considering zero counts,

215 it is also shown in Table 5 that women diagnosed with another tumor type than invasive ductal

216 carcinoma are prone to have no involvement of lymph nodes (OR=6.869, 95\% CI: 5.632-8.376).

217 Patients with tumor grade 1 have positive correlation with not having lymph nodes than grades

$21811(\mathrm{OR}=0.375,95 \%$ CI: 0.309-0.454) and $111(\mathrm{OR}=0.453,95 \%$ CI: 0.372-0.550). ER-negative

219 (OR=0.543，95\% CI: 0.460-0.642), PR-negative (OR=0.277, 95\% CI: 0.229-0.334) and

220 Her2.neu-negative $(\mathrm{OR}=0.429,95 \%$ CI: $0.363-0.508)$ patients have greater probability of no

221 nodal involvement tumor. Tumor size $2-4.9(\mathrm{OR}=0.497,95 \% \mathrm{CI}$ : $0.394-0.626)$ have zero counts 
222 in respect of involved nodes than reference size $\leq 1.9$, and bigger tumor size $\geq 5(\mathrm{OR}=0.036,95 \%$

223 CI: 0.022-0.058).

224

225 -Table 5 -

226

227 Discussion

228 In our data, almost half of the study population with breast cancer showed negative nodal status,

229 which means no involvement of lymph nodes at primary diagnosis of breast cancer. However,

230 this can be due to an early stage of breast cancer which may develop to be more harmful after

231 time passes. Furthermore, there is a probability of human error during data entry. For that reason,

232 choosing the best fit is important in case of accounting false negative. The study population is

233 divided into "at-harm" and "not-at-harm" groups in the context of nodal status, as patients with

234 positive number of involved nodes are considered more critical than those who have no 235 involvement of lymph nodes.

236 We have fitted standard Poisson and negative binomial regression models by incorporating time

237 independent covariates. When it comes to describe the variability of counts, the Poisson model

238 does not fit well, but negative binomial distribution serves a good fit for modeling counts with

239 variability different from mean. This is depicted through the analysis, which resulted into higher

240 AIC values due to ignoring the higher frequency of occurrences in zeroes. Whereas the estimates

241 are correct, standard errors are wrong and unaccounted by the model. Therefore, the results are

242 not displayed in this article.

243 Furthermore, lymph nodes negative status was in greater number than positive ones, which 244 showa a strong evidence of applying zero inflated modeling techniques to get in depth study of 
245 patients' health by analyzing data. Zero inflated models separately analyzed both groups count 246 and excessive zeroes by mixture modeling technique.

247 ZIP is suggested to account excess zeros, but comes with the limitation of equal mean and 248 variance for the count component [34]. Also for heavy tail with extra zero data ZINB model is 249 recommended [29]. In summary, zero-inflated modeling is a better choice as compared to 250 standard regression count models, for the type of study population we have analyzed. Therefore, 251 it is highly recommended that researchers should fit standard and inflated models to choose the 252 best fit for their dataset.

253 In terms of model selection criteria, score and likelihood ratio tests are suitable for comparing 254 nested models [27]. Assuming a well fitted model, the ZINB model is likely to have provided an 255 accurate representation for understudy dataset by AIC non-nested model selection method, a 256 good way to provide tradeoff between complexity of applied model and good fit for study data.

257 Some limitations must be noted. First, the number of covariates added is dependent on the 258 availability of data. Further important factors related to patients not included in this analysis may 259 also affect the nodal status. Second, we were not able to account a longitudinal assessment that 260 may reveal other aspects related to "at-harm" and "not-at-harm" populations. Third, we did not 261 account for different binary components of zero-inflation due to a large number of available 262 covariates. This choice of models needs to be empirically investigated in studies related to breast 263 cancer in the future. Forth, simulation study can be conducted to strengthen our conclusions.

264 Apart from these future tasks, this study is trying to fill the statistical modeling gap to analyze 265 patterns of nodal involvement in primary breast cancer patients, using a large data set collected 266 in Pakistan. Mayo hospital Lahore is one of the best governmental hospital where patients come 267 from all over Pakistan. We believe that our study successfully quantified the "at-harm" 
268 population group by incorporating time independent covariates which are associated with the

269 presence of involved lymph nodes. Zero-inflated models have successfully demonstrated the

270 advantage of fitting count nodal data when both "at-harm" and "not-at-harm" groups are

271 important in predicting disease on set and disease progression.

272

\section{Conclusions}

274 Accurate prediction of involved lymph nodes is a need for clinicians, as it is one of the most

275 important therapeutic and prognostic factors to improve health outcomes of breast cancer

276 patients. Our analysis showed that ZINB is the best model for predicting and describing the

277 number of involved nodes in primary breast cancer, when overdispersion arises due to a large

278 number of patients with no lymph node involvement. Furthermore, advanced tumor grade and

279 larger tumor pose risks, which suggest urgent treatment.

280

281 Abbreviations

282 AIC

Akaike information criterion

283 CI

Confidence interval

284 ER

Estrogen receptor

285 Her2.neu

Human Epidermal Growth Factor Receptor 2

286 OR

Odds ratio

287 PDF

Probability density function

288 PMF

Probability mass function

289 PR Progesterone receptor

290 p-value Probability-value

291 SE

Standard error

292 ZINB

Zero-Inflated Negative Binomial

293 ZIP

Zero-Inflated Poisson 


\section{DECLARATIONS}

\section{Ethics approval and consent to participate}

297 No ethical approval needed, because the study is based on a retrospective analysis of hospital 298 record data.

\section{Consent for publication}

301 Not applicable

\section{Availability of data and materials}

Data is available upon reasonable request from the corresponding author.

\section{Competing interests}

307 The authors declare that no competing interests exist.

\section{Funding}

310 This study received no funding.

\section{Authors' contributions}

313 The study was conceptualized by ML, supported by SK. NZ has been responsible for data 314 acquisition. ML analyzed the data; SK and FF supervised this process. ML drafted the 315 manuscript, SK and FF revised it critically for important intellectual content. All authors 316 approved the final version of the manuscript.

\section{Acknowledgements}

319 We thank the Staff of Oncology and Radiology Department, Mayo Hospital, Lahore, who supported 320 in data collection. We also wish to appreciate Dr. Abbas Khokar (Mbbs, FCPS), Head of Oncology 321 Department from Mayo Hospital, Lahore, Pakistan, for all the efforts to organize patients' records so 322 systematically.

323 We acknowledge support from the German Research Foundation (DFG) and the Open Access 324 Publication Fund of Charité - Universitätsmedizin Berlin. 


\section{Authors' information}

328 Not applicable

\section{References}

1. Bray F, Ferlay J, Soerjomataram I, Siegel RL, Torre LA, Jemal A. Global cancer statistics 2018: GLOBOCAN estimates of incidence and mortality worldwide for 36 cancers in 185 countries. CA Cancer J Clin. 2018;68:394-424.

2. Fan L, Goss PE, Strasser-Weippl K. Current status and future projections of breast cancer in Asia. Breast Care. 2015;10:372-8.

3. Momenimovahed Z, Salehiniya H. Epidemiological characteristics of and risk factors for breast cancer in the world. Breast Cancer. 2019;11:151-64.

4. Liaqat M, Shahid K, Fischer F, Fazil W. Performance Evaluation of Distributional Models to Analyze Random Right Censored Breast Cancer Failure Time Data. BMC Medical Research Methodology [under review].

5. Martin FT, O'Fearraigh C, Hanley C, Curran C, Sweeney KJ. The prognostic significance of nodal ratio on breast cancer recurrence and its potential for incorporation in a new prognostic index. Breast J. 2013;19:388-93.

6. Hong R, Dai Z, Zhu W, Xu B. Association between lymph node ratio and disease specific survival in breast cancer patients with one or two positive lymph nodes stratified by different local treatment modalities. PLoS One. 2015;29:e0138908.

7. Fisher B, Bauer M, Wickerham DL, Redmond CK, Fisher ER, Cruz AB, Foster R, Gardner B, Lerner H, Margolese R, Poisson R, Shibata H, Volk H. Relation of number of positive axillary nodes to the prognosis of patients with primary breast cancer. Cancer. 1983;52(9):1551-7.

8. Nottegar A, Veronese N, Senthil M, Roumen RM, Stubbs B, Choi AH, Verheuvel NC, Solmi M, Pea A, Capelli P, Fassan M, Sergi G, Manzato E, Maruzzo M, Bagante F, Koc M, Eryilmaz MA, Bria E, Carbognin L, Bonetti F, Barbareschi M, Luchini C. Extra-nodal extension of sentinel lymph node metastasis is a marker of poor prognosis in breast cancer patients: A systematic review and an exploratory meta-analysis. Eur J Surg Oncol. 2016;42:919-25. 
9. Rao R, Euhus D, Mayo HG, Balch C. Axillary node interventions in breast cancer: a systematic review. JAMA. 2013;310:1385-94.

10. Ravdin PM, De Laurentiis M, Vendely T, Clark GM. Prediction of axillary lymph node status in breast cancer patients by use of prognostic indicators. Journal of National Cancer Institute. 1994;86(23):1771-5.

11. Olivotto IA, Jackson JSH, Mates D, Andersen S, Davidson W, Bryce CJ, Ragaz J. Prediction of axillary lymph node involvement of women with invasive breast carcinoma a multivariate analysis. Cancer. 1998;83(5):948-55.

12. Tang W, He H, Tu XM. Applied Categorical and Count Data Analysis. 2012. FL: Chapman \& Hall/CRC.

13. Consul PC, Famoye F. Generalized Poisson regression model. Communications in Statistics (Theory \& Method). 1992;2(1):89-109.

14. Hilbe J. Negative Binomial Regression. Cambridge, UK: Cambridge University Press. 2007.

15. Joe H, Zhu R. Generalized Poisson distribution: the property of mixture of Poisson and comparison with negative binomial distribution. Biometrical Journal. 2005;47:219-29.

16. Cox D. Some remarks on overdispersion. Biometrics. 1983;10:269-74.

17. Dean C. Testing for overdispersion in poisson and binomial regression models. Journal of the American Statistical Association. 1992;87:451-7.

18. Cheung YB. Zero-inflated models for regression analysis of count data: a study of growth and development. Stat Med. 2002;21:1461-9.

19. Lambert D. Zero-inflated poisson regression with an application to defects in manufacturing. Technometrics. 1992;34:1-14.

20. Yau KK, Lee AH. Zero-inflated poisson regression with random effects to evaluate an occupational injury prevention programme. Stat Med. 2001;20:2907-20.

21. Agarwal DK, Gelfand A, Citron-Pousty S. Zero-inflated model with application to spatial count data. Environmental and Ecological Statistics. 2002;9:341-55.

22. Hur K, Hedeker D, Henderson W, Khuri S, Daley J. Modeling clustered count data with excess zeros in health care outcomes research. Health Services and Outcomes Research Methodology. 2002;3:5-20. 
23. Rose CE, Martin SW, Wannemuehler KA, Plikaytis BD. On the use of zero-inflated and hurdle models for modeling vaccine adverse event count data. J Biopharm Stat. 2006;16(4):463-81.

24. Buu A, Johnson N, Li R, Tan X. New variable selection methods for zero-inflated count data with applications to the substance abuse field. Stat Med. 2011;30(18):2326-40.

25. Hall DB. Zero-inflated Poisson and binomial regression with random effects: a case study. Biometrics. 2000;56(4):1030-9.

26. Tang W, Lu N, Chen T, Wang W, Gunzler DD, Han Y, Tu XM. On performance of parametric and distribution-free models for zero-inflated and over-dispersed count responses. Stat Med. 2015;34(24):3235-45.

27. Ridout MS, Hinde JP, Demetrio CGB. A score test for testing a zero-inflated Poisson regression model against zero-inflated negative binomial alternatives. Biometrics. 2001;57:219-23.

28. Atkins D, Gallop R. Rethinking how family researchers model infrequent outcomes: A tutorial on count regression and zero-inflated models. Journal of Family Psychology. 2007;21(4):726-35.

29. Yau KK, Wang K, Lee AH. Zero-inflated negative binomial mixed regression modeling of over-dispersed count data with extra zeros. Biom J. 2003;45(4):437-52.

30. Gilthorpe MS, Frydenberg M, Cheng Y, Baelum V. Modelling count data with excessive zeros: The need for class prediction in zero-inflated models and the issue of data generation in choosing between zero-inflated and generic mixture models for dental caries data. Stat Med. 2009;28:3539-53.

31. Vuong, Quang H. Likelihood ratio tests for model selection and non-nested hypotheses. Econometrica. 1989;57(2):307-33.

32. Akaike H. A new look at the statistical model identification. IEEE Transactions on Automatic Control. 1974;19:716-23.

33. R Development Core Team. R: A language and environment for statistical computing. Vienna: R Foundation for Statistical Computing. http://www.R-project.org/. 2011.

34. Yang Z, Hardin JW, Addy CL. Testing overdispersion in the zero-inflated Poisson model. Journal of Statistical Planning and Inference. 2009;139:3340-53. 
Figure 1: Nodal frequency count

421

422

423 Tables

424

425 Table 1: Characteristics of 5,196 patients with primary breast cancer

\begin{tabular}{lc}
\hline & $\mathbf{n}(\%)$ \\
\hline Tumor type & \\
IDC & $2,832(54.5 \%)$ \\
Other & $2,364(45.5 \%)$ \\
Age (in years) & \\
$\leq 35$ & $736(14.2 \%)$ \\
$36-45$ & $1,092(21.0 \%)$ \\
$\geq 46$ & $3,368(64.8 \%)$
\end{tabular}

Tumor grade

1

$2,021(38.9 \%)$

11

$1,618(31.1 \%)$

111

$1,557(30.0 \%)$

\section{Estrogen receptor}

$\begin{array}{ll}\text { Positive } & 2,662(51.2 \%) \\ \text { Negative } & 2,534(48.8 \%)\end{array}$

Progesterone receptor

Positive

2,652 (51.0\%)

Negative

$2,544(49.0 \%)$

\section{Her2.neu receptor}

Positive

Negative

Tumor size (in cm)

$\leq 1.9$

2-4.9

426
$963(18.5 \%)$

$3,650(70.2 \%)$

$2,754(53.0 \%)$

$2,442(47.0 \%)$

$583(11.2 \%)$ 
427 Table 2: Distribution of covariates with respect to positive number of involved nodes

\begin{tabular}{|c|c|c|c|c|}
\hline Positive nodes (n) & $\begin{array}{c}1-2 \\
\text { n }(\%)\end{array}$ & $\begin{array}{c}3-4 \\
n(\%)\end{array}$ & $\begin{array}{c}\geq 5 \\
\mathrm{n}(\%)\end{array}$ & $\begin{array}{l}\text { Total } \\
\text { n }(\%)\end{array}$ \\
\hline \multicolumn{5}{|l|}{ Tumor type } \\
\hline IDC & $412(20.1)$ & $1,010(49.3)$ & $627(30.6)$ & $2,049(100)$ \\
\hline Other & $244(32.9)$ & $314(42.4)$ & $183(24.7)$ & $741(100)$ \\
\hline \multicolumn{5}{|l|}{ Age (in years) } \\
\hline$\leq 35$ & $84(22.2)$ & $181(47.8)$ & $114(30.0)$ & $379(100)$ \\
\hline $36-45$ & $123(20.9)$ & $289(49.1)$ & $177(30.1)$ & $589(100)$ \\
\hline$\geq 46$ & $449(24.6)$ & $854(46.9)$ & $519(28.5)$ & $1,822(100)$ \\
\hline \multicolumn{5}{|l|}{ Tumor grade } \\
\hline 1 & $214(28.2)$ & $458(60.3)$ & $88(11.6)$ & $760(100)$ \\
\hline 11 & $251(25.0)$ & $518(51.6)$ & $235(23.4)$ & $1,004(100)$ \\
\hline 111 & $191(18.6)$ & $348(33.9)$ & $487(47.5)$ & $1,026(100)$ \\
\hline \multicolumn{5}{|l|}{ Estrogen receptor } \\
\hline Positive & $305(21.7)$ & $682(48.4)$ & $421(29.9)$ & $1,408(100)$ \\
\hline Negative & $351(25.4)$ & $642(46.5)$ & $389(28.1)$ & $1,382(100)$ \\
\hline \multicolumn{5}{|c|}{ Progesterone receptor } \\
\hline Positive & $247(19.6)$ & $530(42.1)$ & $481(38.2)$ & $1,258(100)$ \\
\hline Negative & 409 (26.7) & $794(51.8)$ & $329(21.5)$ & $1,532(100)$ \\
\hline \multicolumn{5}{|l|}{ Her2.neu receptor } \\
\hline Positive & $242(21.6)$ & $450(40.2)$ & $427(38.2)$ & $1,119(100)$ \\
\hline Negative & $414(24.8)$ & $874(52.3)$ & $383(22.9)$ & $1,671(100)$ \\
\hline \multicolumn{5}{|l|}{ Tumor size (in cm) } \\
\hline$\leq 1.9$ & $183(73.5)$ & $34(13.7)$ & $32(12.9)$ & $249(100)$ \\
\hline $2-4.9$ & $452(22.8)$ & $1,278(64.4)$ & $253(12.8)$ & $1,983(100)$ \\
\hline$\geq 5$ & $21(3.8)$ & $12(2.2)$ & $525(94.1)$ & $558(100)$ \\
\hline
\end{tabular}


Table 3: Multivariate models to study the effect of time independent covariates on number of involved nodes in 430 primary breast cancer patients

\begin{tabular}{lcc}
\hline Parameter & $\begin{array}{c}\text { Zero-Inflated Poisson (ZIP) } \\
\text { Estimate (SE) [p-value] }\end{array}$ & $\begin{array}{c}\text { Zero-Inflated Negative Binomial (ZINB) } \\
\text { Estimate (SE) [p-value] }\end{array}$ \\
\hline Poisson/NB with log link & $0.512(0.065)[5.39 \mathrm{e}-15]$ & $0.486(0.071)[5.51 \mathrm{e}-12]$ \\
$\begin{array}{l}\text { Intercept } \\
\text { Tumor type }\end{array}$ & Ref. & Ref. \\
$\quad$ IDC & $-0.004(0.025)[0.870]$ & $0.010(0.028)[0.730]$ \\
$\quad$ & & \\
Age (in years) & Ref. & Ref. \\
$\quad 35$ & $-0.006(0.032)[0.861]$ & $0.005(0.037)[0.897]$ \\
$36-45$ & $0.019(0.027)[0.487]$ & $0.019(0.032)[0.540]$
\end{tabular}

Tumor grade

1

11

111
Ref.

$0.006(0.027)$ [0.815]

$0.273(0.026)[<2 \mathrm{e}-16]$
Ref.

$0.002(0.030)$ [0.940]

$0.280(0.030)[<2 \mathrm{e}-16]$

\section{Estrogen receptor}

Positive

Negative

\section{Progesterone receptor}

Positive

Negative

Her2.neu receptor

Positive

Negative

Tumor size (in cm)

$\leq 1.9$

2-4.9

$\geq 5$

$\log ($ theta)

Binomial with logit link

Intercept

Ref.

Ref.

$-0.047(0.019)$ [0.011]

Ref.

$-0.087(0.020)$ [2.07e-05]

Ref.

$-0.027(0.019)$ [0.170]

Ref.

$0.705(0.058)[<2 \mathrm{e}-16]$

$1.622(0.059)[<2 e-16]$

Tumor type

IDC

Other

Age (in years)
Ref.

Ref.
$1.845(0.094)[<2 e-16]$
Ref.

$-0.049(0.021)$ [0.021]

Ref.

$-0.109(0.024)$ [3.91e-6]

$-0.030(0.023)$ [0.178]

Ref.

$0.726(0.061)[<2 \mathrm{e}-16]$

$1.654(0.063)[<2 \mathrm{e}-16]$

$2.908(0.134)[<2 \mathrm{e}-16]$ 


\begin{tabular}{|c|c|c|}
\hline Parameter & $\begin{array}{l}\text { Zero-Inflated Poisson (ZIP) } \\
\text { Estimate (SE) [p-value] }\end{array}$ & $\begin{array}{c}\text { Zero-Inflated Negative Binomial (ZINB) } \\
\text { Estimate (SE) [p-value] }\end{array}$ \\
\hline$\leq 35$ & Ref. & Ref. \\
\hline $36-45$ & $0.073(0.130)[0.573]$ & $0.086(0.134)[0.523]$ \\
\hline$\geq 46$ & $0.027(0.111)$ [0.805] & $0.039(0.115)[0.732]$ \\
\hline \multicolumn{3}{|c|}{ Tumor grade } \\
\hline 1 & Ref. & Ref. \\
\hline 11 & $-0.932(0.093)[<2 \mathrm{e}-16]$ & $-0.982(0.080)[<2 \mathrm{e}-16]$ \\
\hline 111 & $-0.767(0.096)[1.34 \mathrm{e}-15]$ & $-0.792(0.100)[1.82 \mathrm{e}-15]$ \\
\hline \multicolumn{3}{|c|}{ Estrogen receptor } \\
\hline Positive & Ref. & Ref. \\
\hline Negative & $-0.558(0.081)[4.31 \mathrm{e}-12]$ & $-0.610(0.085)[7.48 \mathrm{e}-13]$ \\
\hline \multicolumn{3}{|c|}{ Progesterone receptor } \\
\hline Positive & Ref. & Ref. \\
\hline Negative & $-1.210(0.089)[<2 \mathrm{e}-16]$ & $-1.285(0.096)[<2 \mathrm{e}-16]$ \\
\hline \multicolumn{3}{|c|}{ Her2.neu receptor } \\
\hline Positive & Ref. & Ref. \\
\hline Negative & $-0.828(0.082)[<2 \mathrm{e}-16]$ & $-0.846(0.086)[<2 \mathrm{e}-16]$ \\
\hline \multicolumn{3}{|c|}{ Tumor size (in cm) } \\
\hline$\leq 1.9$ & Ref. & Ref. \\
\hline $2-4.9$ & $-0.728(0.114)[1.74 \mathrm{e}-10]$ & $-0.700(0.118)[3.39 \mathrm{e}-09]$ \\
\hline$\geq 5$ & $-3.362(0.240)[<2 \mathrm{e}-16]$ & $3.320(0.246)[<2 \mathrm{e}-16]$ \\
\hline
\end{tabular}


433 Table 4: Comparison of fitted models by Akaike information criterion (AIC)

\begin{tabular}{ll}
\hline Model & AIC \\
\hline Linear & 23710.43 \\
Poisson & 20777.19 \\
Negative Binomial & 18773.01 \\
Zero-Inflated Poisson & 16658.36 \\
Zero-Inflated Negative Binomial & 16559.15 \\
\hline
\end{tabular}

434 
Table 5: Zero-Inflated Negative Binomial model

\begin{tabular}{lcc}
\hline Parameter & OR & 95\% CI \\
\hline Poisson/NB with log link & 1.626 & $1.416-1.867$ \\
$\begin{array}{l}\text { Intercept } \\
\text { Tumor type }\end{array} \quad 1$ & \\
$\quad$ IDC & 1.010 & $0.955-1.067$ \\
$\quad$ Other & & \\
Age (in years) & 1 & \\
$\quad \leq 35$ & 1.005 & $0.926-1.070$ \\
$36-45$ & 1.019 & $0.958-1.085$ \\
$\quad \geq 46$ &
\end{tabular}

Tumor grade

1 1

11

1.002

$0.944-1.064$

111

1.323

$1.248-1.402$

Estrogen receptor

Positive 1

Negative

0.952

$0.913-0.993$

\section{Progesterone receptor}

Positive

1

Negative

0.897

$0.865-0.939$

\section{Her2.neu receptor}

Positive

1

Negative

0.970

$0.928-1.014$

Tumor size (in cm)

$$
\leq 1.9
$$

1

2-4.9

2.067

$1.836-2.329$

$\geq 5$

5.228

$4.625-5.913$

\section{Binomial with logit link}

Intercept

3.536

$2.527-4.952$

Tumor type

IDC

1

Other

6.869

$5.632-8.376$

Age (in years)

$\leq 35$

1

36-45

1.090

$0.838-1.417$ 


\begin{tabular}{ccc}
\hline Parameter & OR & $\mathbf{9 5 \%}$ CI \\
\hline$\geq 46$ & 1.040 & $0.831-1.302$
\end{tabular}

Tumor grade

1

11

1

111

0.375

$0.309-0.454$

0.453

$0.372-0.550$

Estrogen receptor

Positive

1

Negative

0.543

$0.460-0.642$

Progesterone receptor

Positive

1

Negative

0.277

$0.229-0.334$

Her2.neu receptor

Positive

1

Negative

0.429

$0.363-0.508$

Tumor size (in cm)

$\leq 1.9$

1

2-4.9

0.497

0.394-0.626

$\geq 5$

0.036

0.022-0.058

436 
Figures

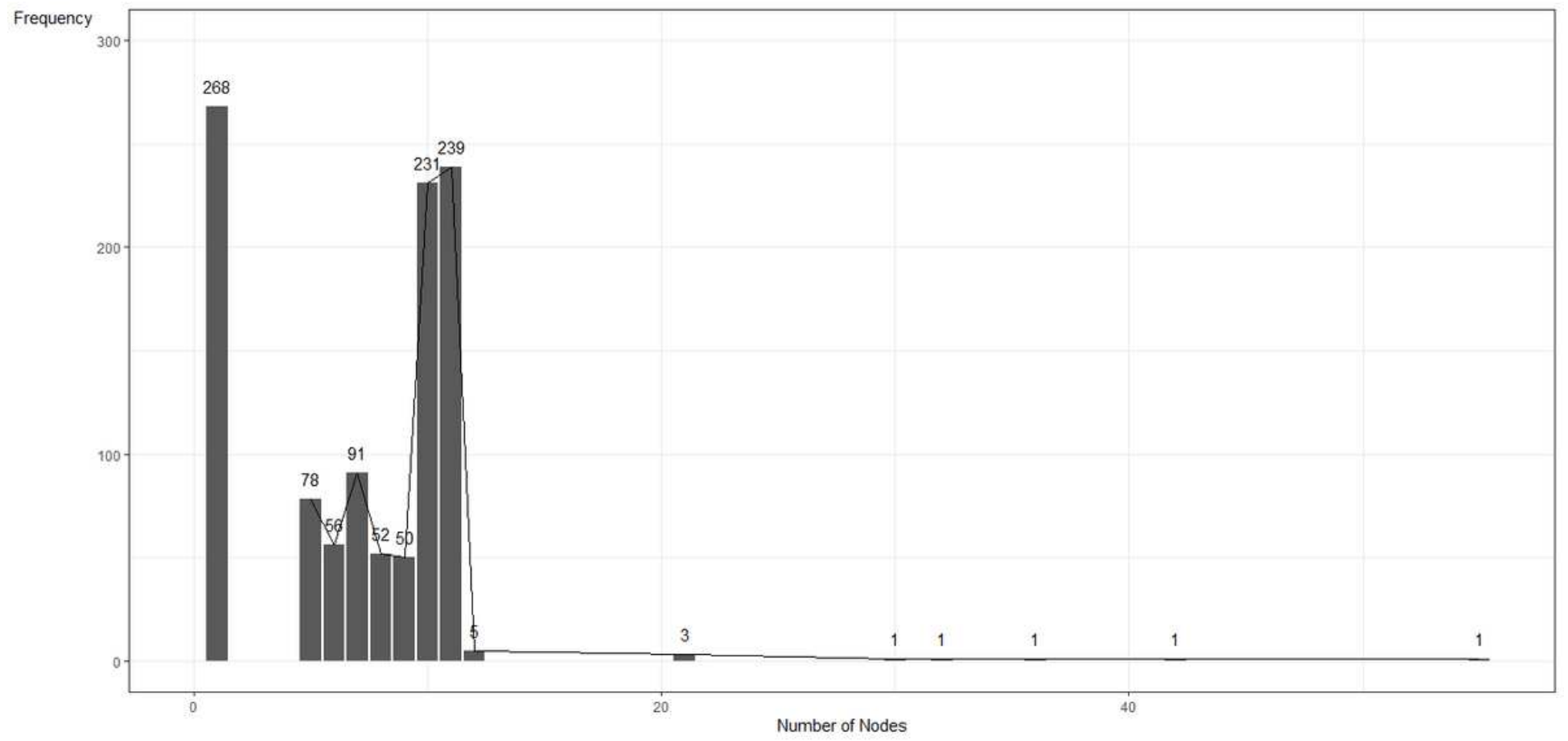

Figure 1

Nodal frequency count 